\title{
The influence of loading rate on the fracture mode for the viscous-elastic composites
}

\author{
I. Viktorova ${ }^{1}$ and S. Alexeeva ${ }^{2}$ \\ ${ }^{1}$ Clemson University, Department of Mathematical Science, 29634 Clemson, SC, USA \\ ${ }^{2}$ Institute of Machine Sciences, 101990, Malyi Kharitonievsky per., 4, Moscow, Russia
}

\begin{abstract}
The study of failure processes of composites should be accompanied with the analysis of the nature of failure, three main categories of which may be identified as: the defect accumulation; macrocracks propagation; instantaneous rupture, being a consequence of the cleavage of a weak link. Usually in these type of investigations the consideration is given to one or another type of failure while the conditions of transition from one type of failure to another or a possible change in its character are not discussed. However, the determination of these conditions is crucial for an accurate analysis of the performance of a composite structural component.
\end{abstract}

Various types of failure for the viscous-elastic composites can be classified into three major categories: 1) the subsequent accumulation of defects, with certain concentration of which resulting in catastrophic structural break; 2) the propagation of the macro cracks or systems of cracks previously occurring in the material; 3) complete and practically instantaneous failure - the result of the cleavage of the weak link. There are multiple publications with consideration given to one or another type of failure while the conditions of transition, i.e., the problem of possible change of the fracture character are not significantly discussed and covered. However, the determination of such conditions for transition is necessary for an accurate analysis of the performance of a structural composite component.

Besides the structural characteristics such as the volume fraction of the fiber [1] that undoubtfully might affect the conditions of the fracture type transition the variable mechanical loading rate as well as temperature are very important factors to consider.

The analysis of the effect of loading rate on the nature of the failure is performed on two types of carbon fibers reinforced composites. The matrix material was the same: epoxyanilinophenol formaldehyde type binder.

As the reinforcement a carbon tape was used with normal (material I) and nitric acid-activated (material II) surfaces. Nitric acid etching promotes an increase in the specific surface (roughness) of the carbon fibers, resulting in the increased shear strength of the materials with treated components by the factor of 1.5-2 [2]. Some physical and mechanical characteristics for both materials are given in table 1 .

The experimental program on tensile tests was carried out at a constant loading rate up to failure in the following range of rates $\left(\mathrm{kg} / \mathrm{mm}^{2} \cdot \mathrm{sec}\right): 6 \cdot 10^{-1}, 2.5 \cdot 10^{0}, 1.25 \cdot 10^{1}$, $1.25 \cdot 10^{2},(6-8) \cdot 10^{2},(1-1.6) \cdot 10^{4}$. The values of the maximum loading were fixed in the experiments and provided the data for the graphs of the dependence of the tensile strength on the loading rate, given in figure 1.The results on durability testing at the constant loading level are given in figure 2 together with the constructed model durability curves.
Analysis of the graphs in figure 1 suggests strongly nonmonotonic character of the dependence of $\sigma_{*}$ on the loading rate. It can be assumed that the occurrence of extrema peaks is due to the change of material failure type.

The first region $\mathrm{AB}$ is due to a progressive accumulation of defects with strength increasing in synch with the loading rate increase. The maximum point for this region corresponds to the moment when the cracks or defects start to grow. To the right from the maximum point the failure takes place by the propagation of the macro cracks and hence is determined by the value of $\mathrm{K}_{I c}$ for the given material, which falls both with the fiber volume content increase and with the rise of the loading rate, illustrated by decreasing region II in figure 1 . The extrema minimum for this decreasing region corresponds to the appearance of cleavage of the weakened link in the composite material resulting in its practically instantaneous failure; from this moment the strength values tend to increase again with the growth of dynamical component of the loading rate.

The developed mathematical model describes the process of damage accumulation in material and allows to establish the dependence of the ultimate breaking stress on time (or the rate) of loading. The failure criterion is formulated as following

$$
\sigma+M^{*} \sigma=\sigma_{0^{*}}
$$

where $\mathbf{M}^{*}$ is hereditary type operator, and $\sigma_{0^{*}}$ is the material constant, corresponding to the limiting value of the cross-sectional stress, which for composites might be taken proportional to the product of the mean strength by the volume fraction for the fibers. With appropriate choice of kernel in (1) the defining equation for the value of the ultimate stress $\sigma_{*}$ and the time to failure for any loading condition is obtained. This model combined with equation (1) is used to describe the rate dependence for $\sigma_{*}$, characteristic for the parts $A_{1} B_{1}$ and $A_{2} B_{2}$ of the diagram in figure 1 as well as to construct the durability curves. For the line segments $A_{1} B_{1}$ and $A_{2} B_{2}$ the parameters for equation (1) were determined based on the kernel taken in the form: $\mathrm{M}(\mathrm{t}-\tau)=\mathrm{m}(1-\alpha) /(\mathrm{t}-\tau)^{\alpha}$. The following 
Table 1. Physical and mechanical characteristics.

\begin{tabular}{|l|r|r|r|}
\hline \multicolumn{1}{|c|}{ Material } & $\begin{array}{c}\text { Binder } \\
\text { 5211-B }\end{array}$ & $\begin{array}{c}\text { Material } \\
\text { I }\end{array}$ & $\begin{array}{c}\text { Material } \\
\text { II }\end{array}$ \\
\hline Density, g/cm ${ }^{3}$ & 1.20 & 1.45 & 1.46 \\
\hline Fiber content $\mathrm{V}_{\mathrm{f}}$, vol. \% & - & 54.00 & 53.50 \\
\hline Pore content $\psi$, vol. \% & - & 4.80 & 2.50 \\
\hline Tensile strength*, kgf $/ \mathrm{mm}^{2}$ & 4.50 & 48.00 & 49.00 \\
\hline Ultimate elongation, \% & 3.60 & 0.63 & 0.60 \\
\hline Elastic modulus, $\mathrm{kgf} / \mathrm{mm}^{2}$ & 260.00 & 7605.00 & 8000.00 \\
\hline Shear strength*, $\mathrm{kgf} / \mathrm{mm}^{2}$ & 4.50 & 2.00 & 4.10 \\
\hline $\mathrm{K}_{I c}, \mathrm{kgf} / \mathrm{mm}^{2}$ & 3.26 & 50.50 & 52.80 \\
\hline
\end{tabular}

* The values were determined under loading with a constant machine grip rate at $\mathrm{v}=2 \mathrm{~mm} / \mathrm{min}\left(\dot{\sigma} \sim 2.5 \mathrm{kgf} / \mathrm{mm}^{2} \cdot \mathrm{sec}\right)$.

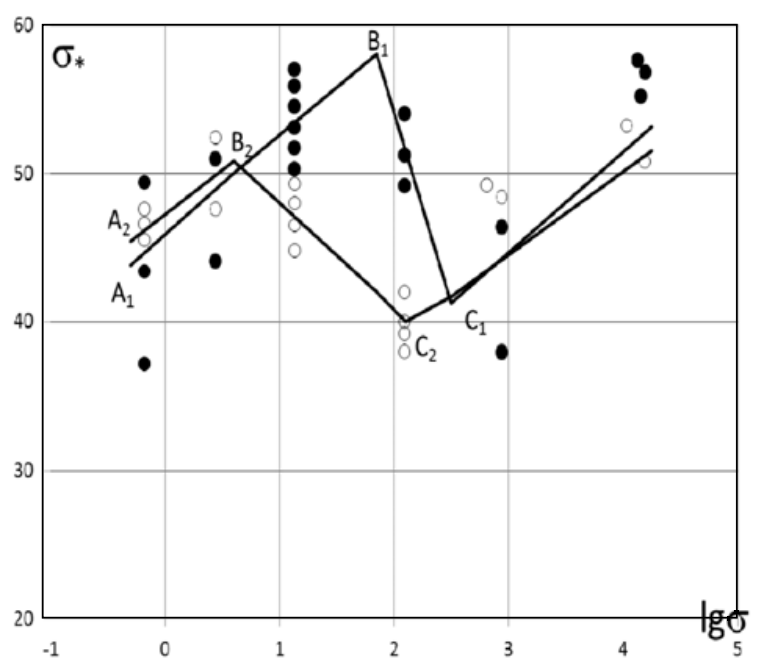

Fig. 1. Tensile strength versus loading rate for two tested materials (I and II). Solid lines - modelled results, dots - experimental data.

values were calculated: for material I, $\alpha=0.92, \mathrm{~m}=$ $0.29 \mathrm{sec}^{-(1-\alpha)}, \sigma_{0^{*}}=65 \mathrm{kgf} / \mathrm{mm}^{2}$; for material II, $\alpha=$ $0.96, \mathrm{~m}=0.946 \mathrm{sec}^{-(1-\alpha)}, \sigma_{0^{*}}=98.3 \mathrm{kgf} / \mathrm{mm}^{2}$.

In figure 1 , the solid line segments $\mathrm{A}_{1} \mathrm{~B}_{1}$ and $\mathrm{A}_{2} \mathrm{~B}_{2}$ represent the estimated results modeled by equation (1) for the loading regime $\dot{\sigma}=$ const. Subsequently the same parameters were used to construct the durability curves (the solid lines in figure 2). It is clear that the estimated set of parameters appear to establish rather good approximation to the available experimental data shown by dots and circles in figure 2 .

A certain disparity in the results obtained for two different materials should be noted. The weak bonding between the fibers and the matrix characteristic for material I tends to facilitate more rapid accumulation of defects and therefore line $\mathrm{A}_{1} \mathrm{~B}_{1}$ (figure 1) proceeds more steeply than $A_{2} B_{2}$ : the same applies also to the analysis of the durability curves (figure 2). On the other hand, the occurrence of weaker adhesion, with corresponding delamination, retards crack propagation and therefore point $\mathrm{B}_{1}$, representing the point of transition from failure due to

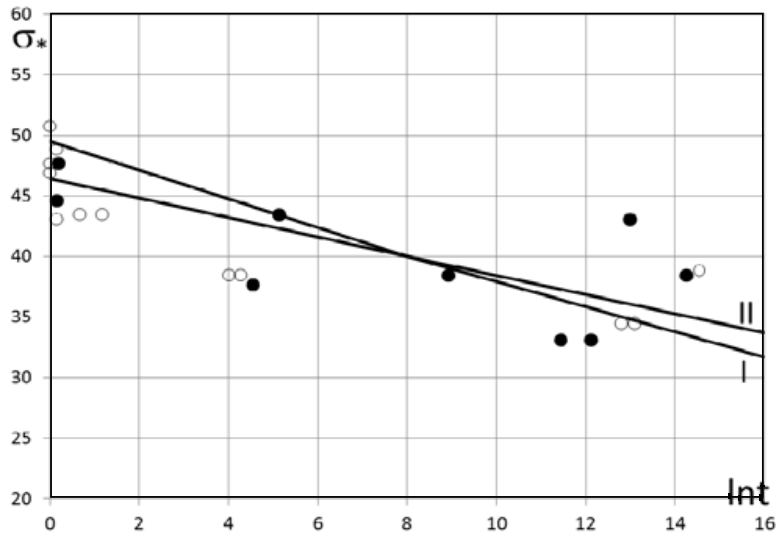

Fig. 2. Durability of two tested materials (I and II). Solid lines modelled results, dots - experimental data.

accumulation of defects to failure from crack propagation, is situated further to the right than is $\mathrm{B}_{2}$.

To analyze the second segment of the rate dependence strength diagram we turn again to equation (1), the righthand side of which has the value $\sigma_{0^{*}}$ characterizing the actual ultimate value proportional to the true stress in the cross section [3].

It is known that if a crack starts to grow, the stress $\sigma$ is related to the intensity coefficient in the following way: $\sigma=K_{I c} / \sqrt{\pi l}$, where $l$ is the size of the crack (or defect). If the material defects do not appear substantially large then, for not too high rates of loading the inequality holds: $\sigma_{0^{*}}<K_{I c} / \sqrt{\pi l}$ (figure 3). This condition also determines the initial section $A_{1} B_{1}\left(A_{2}-B_{2}\right)$ (figure 1). During loading, the value of the true stress in cross section attains its limiting value earlier than appears possible for crack movement. In the case when the rate of loading is quite large $\mathrm{K}_{I c}$ decreases so much that $K_{I c} / \sqrt{\pi l}$ can become equal to or less than $\sigma_{0^{*}}$, hence, this case failure is now determined by the value of $\mathrm{K}_{I c}$. Unfortunately, there was no data available to establish the change of $\mathrm{K}_{I c}$ with the loading rate, therefore the dependences given in figure 3 were calculated for points $\mathrm{B}_{1}$ and $\mathrm{C}_{1}$ and $\mathrm{B}_{2}$ and $\mathrm{C}_{2}$ (figure 1). The values of the loading rates corresponding to the points of transition from defect accumulation to crack propagation, i.e., points $B_{1}$ and $B_{2}$ (figure 1) must correspond to the values $\mathrm{B}_{1}$ and $\mathrm{B}_{2}$ (figure 3 ). Thus, for the conditions when $\sigma_{0^{*}} \leq K_{I c} / \sqrt{\pi l}$, it is necessary to use equation (1), defining the damage accumulation process in order to determine the loading rate dependences of the strength.

Subsequently, for the following stage the equation should be written in the form

$$
\sigma+M^{*} \sigma=\frac{K_{I c}}{\sqrt{\pi l}}
$$

The diagrams in figure 3 are illustrating the dependences of $K_{I c} / \sqrt{\pi l}$ on the loading rate for two studied materials. Values of $\mathrm{K}_{I c}$, shown in table 1 , were determined by the method discussed in [4] at rates of loading of $2.5 \mathrm{kgf} / \mathrm{mm}^{2} \cdot \mathrm{sec}$; in figure 3 they are shown by arrows. By making rather crude assumption of a linear dependence of $K_{I c} / \sqrt{\pi l}$ on $\dot{\sigma}$ it is possible to extend it to the value 


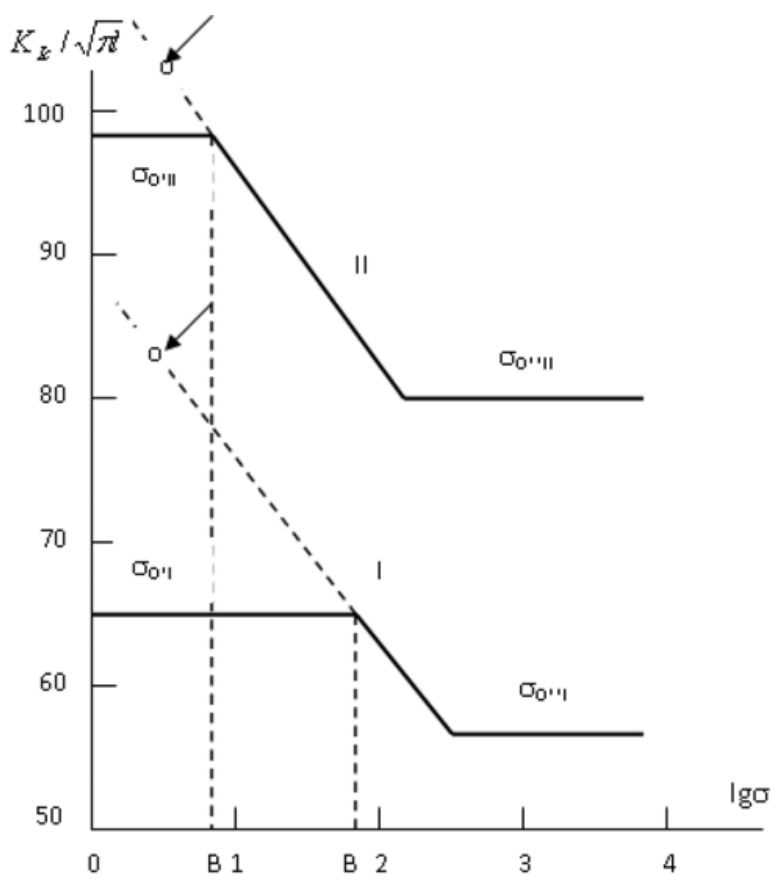

Fig. 3. Determination of parameters for equations (1)-(3).

of $\dot{\sigma}=2.5 \mathrm{kgf} / \mathrm{mm}^{2} \cdot \mathrm{sec}$ and by determining $K_{I c} / \sqrt{\pi l}$ at $\dot{\sigma}=2.5 \mathrm{kgf} / \mathrm{mm}^{2} \cdot \mathrm{sec}$, and by knowing the corresponding values of $\mathrm{K}_{I c}$ taken from table 1, to calculate the approximate sizes of defects for both materials: for material I $l_{\mathrm{I}} \sim 0.1137 \mathrm{~mm}$; material II $l_{\mathrm{II}} \sim 0.0887 \mathrm{~mm}$.

The correctness of these calculations can be verified by means of existing data on the porosity of the material since the ratios of the porosities $\psi_{\mathrm{I}} / \psi_{\mathrm{II}}$ are related to the ratios of the defect sizes (if they are assumed to be spherical, which, of course, can serve only as a very rough approximation) by the relationship $\psi_{\mathrm{I}} / \psi_{\mathrm{I}} \sim l_{I}^{3} / l_{I I}^{3}$. The ratio $\psi_{I} / \psi_{I}=1.92$; the ratio of the sizes $l_{I}^{3} / l_{I I}^{3}=2.1$. Some discrepancy can be explained both by the crudeness of the assumption that the defects are spherical and by insufficient accuracy in determining the porosity and values of $\mathrm{K}_{I c}$.

Thus, equation (2) determines the dependence of the value of the breaking stress on the time to fracture (or on the rate of loading) for sections $B_{1} C_{1}$ and $B_{2} C_{2}$. Since the decrease in the value of $\mathrm{K}_{I c}$ with the rate of loading cannot be infinite, there is apparently a limiting lower critical value $K_{I c} / \sqrt{\pi l}=\sigma_{0^{* *}}$. It can be assumed that if the upper value $\sigma_{0^{*}}$ is determined by the true cross-sectional stress (i.e., by the stress of a material without defects), then the lower limiting value $\sigma_{0^{* *}}$ is to be determined by the nominal stress. Unfortunately, there was no available data on the defectiveness of the cross-sectional area; it will depend not only on the above-determined defect size land on the porosity, but also to a considerable degree on the shape of the defects and their dimensions in the direction of the loading axis. Therefore, the lower limiting value $\sigma_{0^{* *}}$ was determined so as to describe the available experimental data in the best way. The values obtained were: $\sigma_{0^{* *} \mathrm{I}}=56 \mathrm{kgf} / \mathrm{mm}^{2} ; \sigma_{0^{* *} \mathrm{II}}=81 \mathrm{kgf} / \mathrm{mm}^{2}$.
The corresponding failure criterion can be obtain from equation (1) by substituting the lower limiting value into the right -hand side and thus obtaining:

$$
\sigma+M^{*} \sigma=\sigma_{0^{* *}}
$$

The calculated dependences of the ultimate stress on the rate of loading are shown in figure 1 by solid lines.

It should be emphasized that for all considered cases the left-hand sides of the equations (1)-(3) are the same, the parameters of the operator $M^{*}$ depend only on the properties of the components and the structure of the material, while the right-hand sides are defined by the dependences, represented in figure 3, which are determined by the change in the nature of the failure of the composite, affected by the loading rate.

\section{Conclusions}

The obtained results demonstrate that both studied materials exhibit strongly nonmonotonic dependence of the strength on the loading rate; the occurrence of extrema is due to the change of the failure mechanism type. A mathematical formulation of the strength criterion can be suggested in the form: $\sigma+M^{*} \sigma=\tilde{\sigma}_{0}$, where $\tilde{\sigma}_{0}=\sigma_{0^{*}}$ for $\sigma_{0^{*}} \leq K_{I c} / \sqrt{\pi l} ; \tilde{\sigma}_{0}=K_{I c} / \sqrt{\pi l}$ for $\sigma_{0^{* *}} \leq K_{I c} / \sqrt{\pi l} \leq$ $\sigma_{0^{*}} ; \tilde{\sigma}_{0}=\sigma_{0^{* *}}$ for $K_{I c} / \sqrt{\pi l} \leq \sigma_{0^{* *}}$.

By comparing materials I and II, which contain the same components in the same proportions and which possess practically the same values of static strength, it is evident that under different conditions they behave differently. As indicated earlier, the poorer bonding between the fiber and matrix of material I facilitates the occurrence of delamination, which hinders crack propagation, therefore, point $\mathrm{B}_{1}$ corresponds to a higher rate of load application than point $\mathrm{B}_{2}$. Hence, it can be concluded that for the shortterm applications of the structural components subject to a single-stage loading the preference should be given to material I - due to the fact that delamination dissipates impact energy and the value of the ultimate stress is increased approximately by $30 \%$ with the loading rate increase by two orders of magnitude. However, under the repeated cyclic loading conditions or a prolonged period under load, material I would be worse than material II, since the accumulation of defects in it proceeds more intensely; this follows from the steeper slope of section $\mathrm{A}_{1} \mathrm{~B}_{1}$ compared with $\mathrm{A}_{2} \mathrm{~B}_{2}$ and from a comparison of the slopes of the durability curves.

\section{References}

1. S.T Mileiko. Composite Materials, 274-285 (1979)

2. L.P. Kobets, M.A. Kuznetsova, and G.M. Gunyaev. Plast. Massy. 9 (1976)

3. Yu.V. Suvorova. Mekh. Tverdogo Tela. 4, 107-111 (1979)

4. J.E. Shrooley. Fracture. 4, 50-54 (1977) 